\title{
Expanded Disability Status Scale (EDSS) estimation in Multiple Sclerosis from posturographic data
}

Hua Cao ${ }^{\mathrm{a}, \mathrm{b}, \mathrm{c}^{*}}$, Laurent Peyrodie ${ }^{\mathrm{a}, \mathrm{b}, \mathrm{c}, \mathrm{d}}$, Samuel Boudet ${ }^{\mathrm{a}, \mathrm{b}, \mathrm{e}}$, Fabrice Cavillon ${ }^{\mathrm{a}, \mathrm{b}, \mathrm{e}}$, Olivier Agnani ${ }^{\mathrm{a}, \mathrm{b}, \mathrm{e}, \mathrm{f}}$, Patrick Hautecoeur ${ }^{\mathrm{a}, \mathrm{b}, \mathrm{e}, \mathrm{g}}$, Cécile Donzé $\mathrm{e}^{\mathrm{a}, \mathrm{b}, \mathrm{e}, \mathrm{f}}$

${ }^{a}$ Univ Nord de France, F-59000 Lille, France.

${ }^{b}$ UCLille, F-59000 Lille, France.

${ }^{c}$ Hautes Etudes d'Ingénieur (HEI), Unité de Traitement des Signaux Biomédicaux (UTSB), Lille, France.

'Université de Lille 1, Laboratoire d'Automatique et Génie Informatique et Signal(LAGIS), Lille, France.

${ }^{e}$ Groupe Hospitalier de l'Institut Catholique Lillois / Faculté Libre de Médecine, F-59000 Lille, France.

${ }^{f}$ Physical medicine and Rehabilitation department, Hôpital Saint-Philibert, Lomme, France. ${ }^{g}$ Neurology department, Hôpital de Saint-Vincent, Lomme, France. 


\section{ABSTRACT}

Expanded disability status scale (EDSS) is the most widely used clinical scale to evaluate levels of multiple sclerosis (MS). As MS can lead to disruptions in the regulation of balance and the disability can be evaluated by force platform posturography, we have developed in this study a new strategy to estimate EDSS from postural data. 118 volunteers with EDSS ranging from 0 to 4.5 participated in this study, with eyes closed. By using second-order polynomial regression models, EDSS was estimated from two postural sway parameters, respectively, the length and the surface and four recurrence quantification analysis (RQA) parameters: percentage of recurrence (\%Rec), Shannon entropy (Ent), mean diagonal line length (LL) and trapping time (TT). In addition, all four RQA parameters were calculated for position, instantaneous velocity and acceleration of the center of pressure. In order to select the most accurate method for estimating EDSS, four statistical indices (percentage of agreement, underestimation and overestimation, as well as mean error) were calculated comparing clinical and estimated EDSS scores. The results demonstrate that estimations of EDSS from surface, \%Rec and LL of position, best agreed with clinical scores. This study emphasizes the possibility of distinguishing EDSS scores using postural sway and RQA parameters.

\section{Introduction}

Multiple sclerosis (MS) is a chronic inflammatory and immunological disease characterized by demyelization of the central nervous system [1]. It is one of the most frequent neurological diseases in young adults [2] and its prevalence shows a heterogeneous distribution amongst countries [3]. Symptoms of MS are multifold, and varied including visual disturbances, pyramidal signs (muscle weakness and spasticity), sensory dysfunction, incoordination, balance disorders and cognitive impairment.

Over the course of the disease, MS can lead to severe handicap. To evaluate the degree of neurologic impairment in MS, Kurtzke [4] proposed the expanded disability status scale (EDSS), which is the most widely used scale. EDSS is a 20-step scale of disease severity ranging from 0 (normal) to 10 (death due to MS). This scale includes two parts: one (from 0 to 3.5) taking into account functional parameters and the other (from 4 to 10 ) estimating degrees of mobility in patients. The scale considers eight functional systems (FS): pyramidal, cerebellar, brainstem, sensory, bowel \& bladder, visual, cerebral, and other. These FS represent eight different areas of the central nervous system and each ranges from 0 (normal) to 5 or 6 (maximal impairment). These FS grades, indications of mobility and restrictions in daily life, are used to define EDSS as an ordinal measurement whereby differences between scale steps are not homogeneous. In order to overcome the neurologist interexamination variability, a minimum of 1.0 EDSS unit difference has been needed to define a significant clinical change [5]. Although EDSS has some shortcomings, it is still considered as the standard scale for quantitative assessment of the MS status.

Perturbations in balance representing one of the main symptoms in MS Sensory disorders, appear early in the disease and considerably hamper patients. Proprioceptive ataxia is found in more than $80 \%$ of cases after 5 years of evolution and contributes to increase balance disturbances [6]. Force platform posturography is a common method for quantifying balance performance. Various parameters derived from the center of pressure (COP) signal provide different types of information on postural control mechanisms [7]. Displacements of the COP in anteroposterior and/or mediolateral directions are used in the analysis of postural sway [8, 9]. Recurrence quantification 
analysis (RQA) has been presented in order to provide many features of given time series, including the quantification of deterministic structures and of non stationarity [10].

Some studies $[2,11]$ have used a force platform to evaluate balance control in MS patients observing significantly greater sway (postural disorders) in the MS group than in controls. In a previous paper [12], we found a significant correlation between EDSS and posturologic data. Hence, the purpose of the present study was to estimate EDSS scores by using values extracted from the posturologic data of MS patients, in order to select the most pertinent parameters.

\section{Material and Methods}

\subsection{Participants}

89 MS patients and 29 healthy subjects were included in this study (Table 1). Ms Patients were selected among those who underwent neurological consultation at Saint Philibert Hospital, Lomme, France. Healthy controls were recruited from the hospital staff. The diagnosis of MS based on clinical, biological and magnetic resonance imaging findings was established under carefully standardized conditions, in accordance with MacDonald criteria revised in 2005 [13]. The EDSS score was set by a well-trained neurologist with extensive clinical experience. For the patients, the median EDSS score was 3 with a range between 1 and 4.5 .

Inclusion criteria were age: $18-70$ years and relapsing remittant MS range: 0 to 4.5 .

Exclusion criteria were mental disorders affecting comprehension, orthopedic or rheumatologic perturbation within six months prior to inclusion for all and, at least three months after a recognized relapse for MS. Pyramidal, sensory, visual, cerebellar and brainstem impairments which can alter balance regulation were thereby listed (presence or absence) for each patient. Disturbance data of patients for each EDSS score are presented in Table 2. Written consent before study onset had been obtained from all participants.

\subsection{Study protocol}

Patients were asked to stand upright on a force plate with bare feet and with arms by their sides. They were instructed to stand as still as possible during the record. A Satel force plate was used in our experiments. This balance analysis technique is based on the measurement of COP sway in a standing subject and enables calculation of the intensity and vector of ground reaction forces. Recording time was 51.2 seconds with a sampling frequency of $40 \mathrm{~Hz}$. The evaluation was performed under static conditions with eyes closed. COP variability is generally higher under eyes-closed conditions than under eyes-open conditions, for the same subject, in quiet stance [2]. Records were directed and controlled by a physiotherapist specialized in clinical posturology.

\subsection{Measures for estimating EDSS score}

\subsubsection{Postural sway measures}

Two estimates of postural sway were computed for each trial: length, which represents the total length of the COP path over time, and surface, which corresponds to the surface area of the ellipse that circumscribed $90 \%$ of COP points, computed by principal component analysis (PCA). 


\subsubsection{RQA measures}

RQA is a tool for studying the temporal dynamics of a time series by providing quantitative information on the properties of the underlying dynamic process $[9,14]$. It is based on the construction of a recurrence plot (RP) from which quantitative values are extracted.

Due to COP fluctuations of the subject with eyes closed forming a dynamic structure, RQA was first performed by using the coordinate position $(X, Y)$ of all COP points. Four RQA estimates were calculated from a constructed RP [10]: (1) percentage of recurrence (\%Rec) which is expressed as the density of recurrence points, (2) Shannon entropy (Ent) which represents the probability of finding a diagonal of a given length, (3) mean diagonal line length (LL) which measures the mean length of the diagonal lines, and (4) trapping time (TT) which quantifies the mean length of the vertical lines. For these calculations, the time delay was set to $1 / 40$ s and the embedding dimension was 1 . The minimal diagonal length was set to 2 samples. The radius threshold for identify recurrence was set to $15 \mathrm{~mm}$ for maximum correlation between EDSS and each recurrence estimate.

To further explore the dynamics of the posturogram which reflects the movement of the COP, two movement parameters, instantaneous velocity $V(i)$ and acceleration $A(i)$, were analyzed by using RQA and calculated as follows:

$$
\begin{aligned}
& V(i)=\sqrt{\left(V_{x}(i)\right)^{2}+\left(V_{y}(i)\right)^{2}}, \\
& A(i)=\sqrt{\left(A_{x}(i)\right)^{2}+\left(A_{y}(i)\right)^{2}},
\end{aligned}
$$

where $\quad V_{x}(i)=(X(i+1)-X(i-1)) /(2 * \Delta t) \quad, \quad V_{y}(i)=(Y(i+1)-Y(i-1)) /(2 * \Delta t)$, $\left.A_{x}(i)=\left(V_{x}(i+1)-V_{x}(i)\right) /(\Delta t), A_{y}(i)=\left(V_{y}(i+1)-V_{y}(i)\right) /(\Delta t)\right), \Delta$ t was the sample duration equal to $1 / 40 \mathrm{~s}, V(i)$ and $A(i)$ were in $\mathrm{mm} / \mathrm{s}$ and $\mathrm{mm} / \mathrm{s}^{2}$ respectively. Except for the radius value (39 $\mathrm{mm} / \mathrm{s}$ for the velocity and $360 \mathrm{~mm} / \mathrm{s}^{2}$ for the acceleration), all other parameters were as mentioned previously.

\subsection{Analysis}

Correlation coefficients were calculated between clinical EDSS scores and the logarithm of each calculated estimate. If correlation was significant $(p<0.05)$, a second-order polynomial regression was applied to obtain an equation. EDSS scores were then established by using the estimate and the equation. In order to select the most pertinent value for estimating EDSS score, four statistical indices between clinical and estimated EDSS scores were determined for each estimate: percentage of agreement within a \pm 0.5 EDSS steps (\% Agreement), underestimation, i.e. the estimated score was lower than the clinical score (\% Underestimation) and overestimation, i.e. the estimated score was higher than the clinical score (\% Overestimation). In addition, the mean of the estimation errors was calculated (i.e. the absolute value of the difference between the estimated and clinical values for each participant (Mean error).

\section{Results}


Figs. $1 \mathrm{~A}$ and $1 \mathrm{~B}$ present, respectively, the distributions of instantaneous velocity and acceleration for each EDSS score. In all cases, excepting perhaps at EDSS=4, both velocity and acceleration increase significantly with EDSS score (ANOVA, $p<0.05$ ).

Figs. $2 \mathrm{~A}$ and $2 \mathrm{~B}$ plot clinical EDSS scores as a function of the logarithm of the surface (Fig. 2A) and of \%Rec of the position (Fig. 2B) with the corresponding second-order polynomial regressions and equations. EDSS scores increase with the surface log and decrease with \%Rec.

Correlation coefficients (R) between clinical EDSS scores and all estimates are shown in Table 3. The four statistical indices (\%Agreement, \%Underestimation, \%Overestimation and Mean error) calculated comparing clinical EDSS scores and EDSS scores estimated from postural sway and from the RQA of the position, the velocity and the acceleration are also reported in Tables $3 \mathrm{~A}$ and $3 \mathrm{~B}$ respectively. All correlations were found to be significant $(p<0.05)$, although the highest correlation was between the Ent of the position and the clinical EDSS score $(R=-0.8448)$.

On the basis of highest \%Agreement and lowest mean error, best results were obtained with \%Rec of the position (\%Agreement $=70.49 \%$, Mean error $=0.63$ ), surface (\%Agreement $=68.03 \%$, Mean error $=0.62$ ) and LL of the position (\%Agreement $=68.03 \%$, Mean error $=0.63$ ).

The difference between \%Overestimation and \%Underestimation ranged from $-4.10 \%$ (\%Rec of the velocity) to $3.28 \%$ (Ent of the position).

\section{Discussion}

Our cohort disability level was similar to other MS published cohorts [6, 15]. Pyramidal, sensory and visual impairments are usually present in early stages of the disease while cerebellar involvement generally occurs later on [15].

EDSS is the most widely used clinical scale for evaluating disability in people with MS. It is usually determined by the physician specialist who performs the neurological examination. Although some methods have been developed for the automatic estimation of EDSS, most are based on a set of predefined rules derived from functional systems findings $[16,17]$. In this study, we extracted two postural sway estimates and four RQA values from posturologic data, all having a significant correlation with the clinical EDSS scores. This observation corroborates and extends our previous study [12] in which only the two parameters of surface of the circumscribing ellipse and \%Rec of the COP position were considered. It is thus possible to estimate EDSS scores from the posturologic data with a Mean error of less than 0.85 .

Furthermore, during a previous study, we presented an EDSS estimation method by using RQA measures of COP position [12]. In order to provide a more detailed view of the dynamics of COP trajectory, we herein proposed to calculate RQA values of instantaneous velocity and acceleration of the COP and expected that these two movement parameters would more accurately estimate EDSS scores compared to COP position. However, the results show that the RQA parameters of position are generally more discriminant than those of velocity and acceleration.

The first possible reason is that recurrence measurements of instantaneous velocity and acceleration require a higher sampling rate than that to sample the trajectory of the COP. The 
recurrence method used in this study to analyze the combined $X-Y$ data has a combined maximum frequency which may be higher than that for each individual coordinate. What is more, as taking the derivative of a signal (velocity and acceleration) attenuates lower frequency components and amplifies higher frequency components of a signal, a higher sampling rate is necessary to reproduce the signal [18]. For this, we propose to increase the sampling rate to $100 \mathrm{~Hz}$ in agreement with previous authors as [19] and then to filter COP signals with a low-pass filter before calculating velocity and acceleration. This will decrease the sensitivity to noise ratio by which the numerical derivatives are easily affected.

The second possible explanation is based on the profile of the instantaneous velocity and acceleration over the EDSS scale (Figs. 1A and 1B). Except for EDSS=4, both velocity and acceleration increase significantly with EDSS score. It is well known that lower scores, up to 3.5 , are largely dependent on the functional systems, but disability in the higher scores is mainly determined in reference to ambulation. EDSS is a mixture of impairment (lower EDSS levels) and disability (higher EDSS levels) heavily weighted towards ambulation. Therefore, presence of different pathological behaviors at EDSS $=4$ could affect the representation of the posturogram and the profile of the velocity and acceleration, such as spasticity [20].

\section{Conclusion}

In this study, we presented methods for estimating EDSS score of patients with MS, from postural data. The most pertinent and accurate method uses surface of the ellipse, \%Rec and LL of the COP position. The results reinforce the ability to well estimate EDSS scores and further the possibilities distinguishing EDSS scores by using postural sway and RQA values. In the future, the combination of these parameters, the examination of other values such as time delay and embedding dimensions for construction of the RP matrix and the use of shorter intervals of a few minutes or a few days may prove fruitful.

\section{Acknowledgment}

The authors would like to thank the Saint-Philibert Hospital, Lille, for providing postural data of the healthy subjects and the patients with multiple sclerosis, and for proofreading of the manuscript, Dr. David F. Perry.

\section{References}

[1] Storch J, Lassmann H. Pathology and pathogenesis of demyelinating diseases. Curr Opin Neurol 1997; 10: 186-92.

[2] Yahia A, Ghroubi S, Mhiri C, Elleuch MH. Relationship between muscular strength, gait and postural parameters in multiple sclerosis. Ann Phys Rehabil Med 2011; 54: 144-55.

[3] Rosati G. The prevalence of multiple sclerosis in the world: an update. Neurol Sci 2001; 22: 11739.

[4] Kurtzke JF. Rating neurologic impairment in multiple sclerosis: an expanded disability status scale (EDSS). Neurology 1983; 33: 1444-52.

[5] Sharrack B, Hughes RA. Clinical scales for multiple sclerosis. J Neurol Sci 1996; 135: 1-9. 
[6] Weinshenker BG. Epidemiology of multiple sclerosis. Neurol Clin 1996; 14: 291-308.

[7] Palmieri RM, Ingersoll CD, Stone MB, Krause BA. Center-of-pressure parameters used in the assessment of postural control. J Sport Rehabil 2002; 11: 51-66.

[8] Riley MA, Clark S. Recurrence analysis of human postural sway during the sensory organization test. Neurosci Lett 2003; 342: 45-8.

[9] Seigle B, Ramdani S, Louis Bernard P. Dynamical structure of center of pressure fluctuations in elderly people. Gait Posture 2009; 30: 223-6.

[10] Riley MA, Balasubramaniam R, Turvey MT. Recurrence quantification analysis of postural fluctuations. Gait Posture 1999; 9: 65-78.

[11] Porosinska A, Pierzchala K, Mentel M, Karpe J. Evaluation of postural balance control in patients with multiple sclerosis - effect of different sensory conditions and arithmetic task execution. A pilot study. Neurol Neurochir Pol 2010; 44: 35-42.

[12] Peyrodie L, Boudet S, Pinti A, Cavillon F, Agnani O, Gallois P. Relations entre posturologie et score EDSS. Sciences et Technologies pour le Handicap 2010; 4: 55-71.

[13] Polman CH, Reingold SC, Edan G, Filippi M, Hartung H, Kappos L, Lublin FD, Metz LM, McFarland HF, O'connor PW, SandBerg-Wolleim M, Thompson AJ, Weinshenker BG, Wolinsky JS. Diagnostic criteria for multiple sclerosis: 2005 revisions to the MacDonald. Ann Neurol 2005; 58: 840-6.

[14] Mazaheri M, Salavati M, Negahban H, Sanjari MA, Parnianpour M. Postural sway in low back pain: Effects of dual tasks. Gait Posture 2010; 31: 116-21.

[15] Confavreux C, Aimard G, Devic M. Course and prognosis of multiple sclerosis assessed by the computerized data processing of 349 patients. Brain 1980; 103: 281-300.

[16] GaspariM, Roveda G, ScandellariC, StecchiS. An expert system for the evaluation of EDSS in multiple sclerosis. Artif Intell Med 2002; 25: 187-210.

[17] Cheng EM, Hays RD, Myers LW, Ellison GW, Beckstrand M, Vickrey BG. Factors related to agreement between self-reported and conventional Expanded Disability Status Scale (EDSS) scores. Mult Scler 2001; 7: 405-10.

[18] Goldberger JJ, Ng J. Practical signal and image processing in clinical cardiology. Springer London Ltd, 2010.

[19] Cornilleau-Pérès V, Shabana N, Droulez J, Goh JCH, Lee GSM, Chew PTK. Measurement of the visual contribution to postural steadiness from the COP movement: methodology and reliability. Gait Posture 2005; 22: 96-106.

[20] Sosnoff JJ, Shin S, Motl RW. Multiple sclerosis and postural control: the role of spasticity. Arch Phys Med Rehabil 2010; 91: 93-9. 
Fig. 1. Instantaneous velocity (A) and acceleration (B) for each EDSS score (ANOVA, $p<0.05$ ).

Fig. 2. EDSS score as a function of the logarithm of the surface (A) and of the \%Rec of the position (B). 
Table 1

Demographic data.

\begin{tabular}{|c|c|c|c|}
\hline & \multirow{2}{*}{$\begin{array}{c}\text { Control } \\
\mathrm{n}=29(\mathrm{~m} / \mathrm{w}=8 / 21) \\
\text { Age }(\mathrm{y})\end{array}$} & \multicolumn{2}{|c|}{$\begin{array}{c}\text { MS Group } \\
\mathrm{n}=89(\mathrm{~m} / \mathrm{w}=20 / 69)\end{array}$} \\
\hline & & Age $(y)$ & Diagnostic Duration (y) \\
\hline Mean & 34.79 & 46.11 & 13.46 \\
\hline SD & 12.31 & 10.26 & 8.89 \\
\hline
\end{tabular}

n: number; m/w: sex ratio. 
Table 2

Disturbances in MS patients for each EDSS score.

\begin{tabular}{ccccccc}
\hline EDSS score & $\mathrm{n}$ & Pyramidal & Sensory & Visual & Cerebellar & Brainstem \\
\hline 1 & 5 & 2 & 4 & 3 & 0 & 0 \\
1.5 & 16 & 5 & 14 & 7 & 1 & 2 \\
2 & 12 & 4 & 12 & 6 & 1 & 0 \\
2.5 & 11 & 11 & 11 & 4 & 1 & 3 \\
3 & 14 & 14 & 14 & 3 & 2 & 7 \\
3.5 & 12 & 7 & 12 & 4 & 3 & 5 \\
4 & 11 & 8 & 11 & 2 & 3 & 3 \\
4.5 & 8 & 8 & 8 & 2 & 3 & 5 \\
\hline
\end{tabular}

n: number. 


\section{Table 3}

Statistical analysis: (A) postural sway measures and (B) RQA measures of the COP's position, instantaneous velocity and instantaneous acceleration.

\begin{tabular}{lll}
\hline & Surface & Length \\
\hline $\mathrm{R}$ & $0.8446^{*}$ & $0.8335^{*}$ \\
\%Agreement & $68.03 \%$ & $63.12 \%$ \\
\%Underestimation & $17.21 \%$ & $18.03 \%$ \\
\%Overestimation & $14.76 \%$ & $18.85 \%$ \\
Mean error & 0.62 & 0.68 \\
\hline
\end{tabular}

(A)

\begin{tabular}{|c|c|c|c|c|}
\hline & & Position & Velocity & Acceleration \\
\hline \multicolumn{5}{|l|}{ \%Rec } \\
\hline & $\mathrm{R}$ & $-0.8324 *$ & $-0.8004^{*}$ & $-0.7221 *$ \\
\hline & \%Agreement & $70.49 \%$ & $64.76 \%$ & $59.84 \%$ \\
\hline & $\%$ Underestimation & $15.57 \%$ & $19.67 \%$ & $19.67 \%$ \\
\hline & \%Overestimation & $13.94 \%$ & $15.57 \%$ & $20.49 \%$ \\
\hline & Mean error & 0.63 & 0.72 & 0.77 \\
\hline \multicolumn{5}{|l|}{ Ent } \\
\hline & $\mathrm{R}$ & $-0.8448 *$ & $-0.7853^{*}$ & $-0.7427^{*}$ \\
\hline & \%Agreement & $65.58 \%$ & $58.20 \%$ & $54.10 \%$ \\
\hline & \%Underestimation & $15.57 \%$ & $21.31 \%$ & $22.13 \%$ \\
\hline & \%Overestimation & $18.85 \%$ & $20.49 \%$ & $23.77 \%$ \\
\hline & Mean error & 0.65 & 0.76 & 0.84 \\
\hline \multicolumn{5}{|l|}{ LL } \\
\hline & $\mathrm{R}$ & $-0.8214^{*}$ & $-0.7532 *$ & $-0.6863 *$ \\
\hline & \%Agreement & $68.03 \%$ & $53.28 \%$ & $54.92 \%$ \\
\hline & $\%$ Underestimation & $15.58 \%$ & $23.77 \%$ & $22.13 \%$ \\
\hline & \%Overestimation & $16.39 \%$ & $22.95 \%$ & $22.95 \%$ \\
\hline & Mean error & 0.63 & 0.77 & 0.82 \\
\hline \multicolumn{5}{|l|}{ TT } \\
\hline & $\mathrm{R}$ & $-0.8123 *$ & $-0.7513^{*}$ & $-0.6907^{*}$ \\
\hline & \%Agreement & $65.58 \%$ & $54.92 \%$ & $55.74 \%$ \\
\hline & $\%$ Underestimation & $17.21 \%$ & $23.77 \%$ & $22.95 \%$ \\
\hline & \%Overestimation & $17.21 \%$ & $21.31 \%$ & $21.31 \%$ \\
\hline & Mean error & 0.65 & 0.77 & 0.81 \\
\hline
\end{tabular}

(B)

R: correlation coefficient between clinical EDSS scores and calculated measures; *: statistically significant correlation, $\mathrm{p}<0.05$; \%Agreement, \%Underestimation and \%Overestimation: percentages of agreement with a \pm 0.5 EDSS steps allowed, underestimation and overestimation between estimated and clinical EDSS scores; Mean error: mean of the estimation errors for each participant. 


\section{Figure1}

Click here to download high resolution image

A

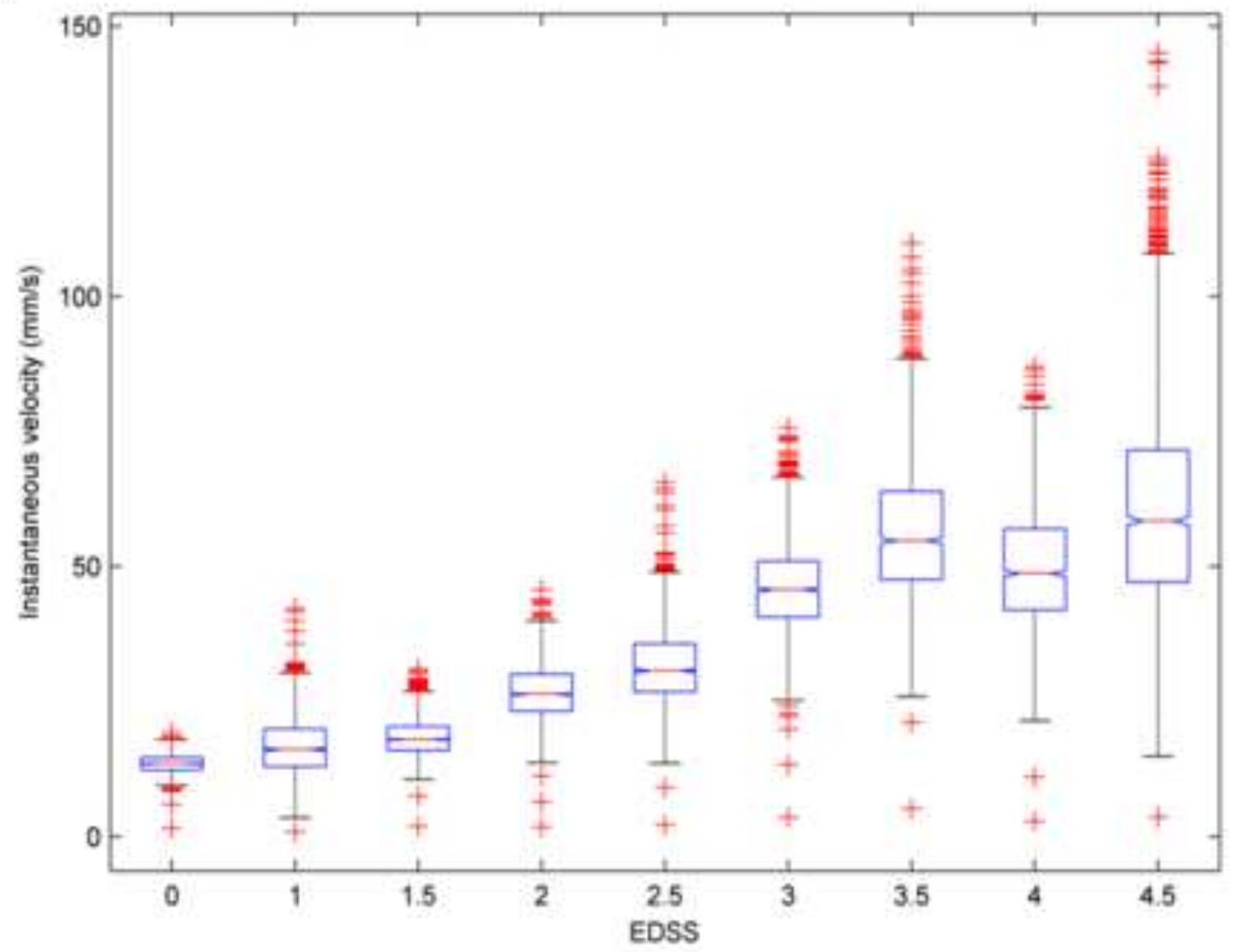

B

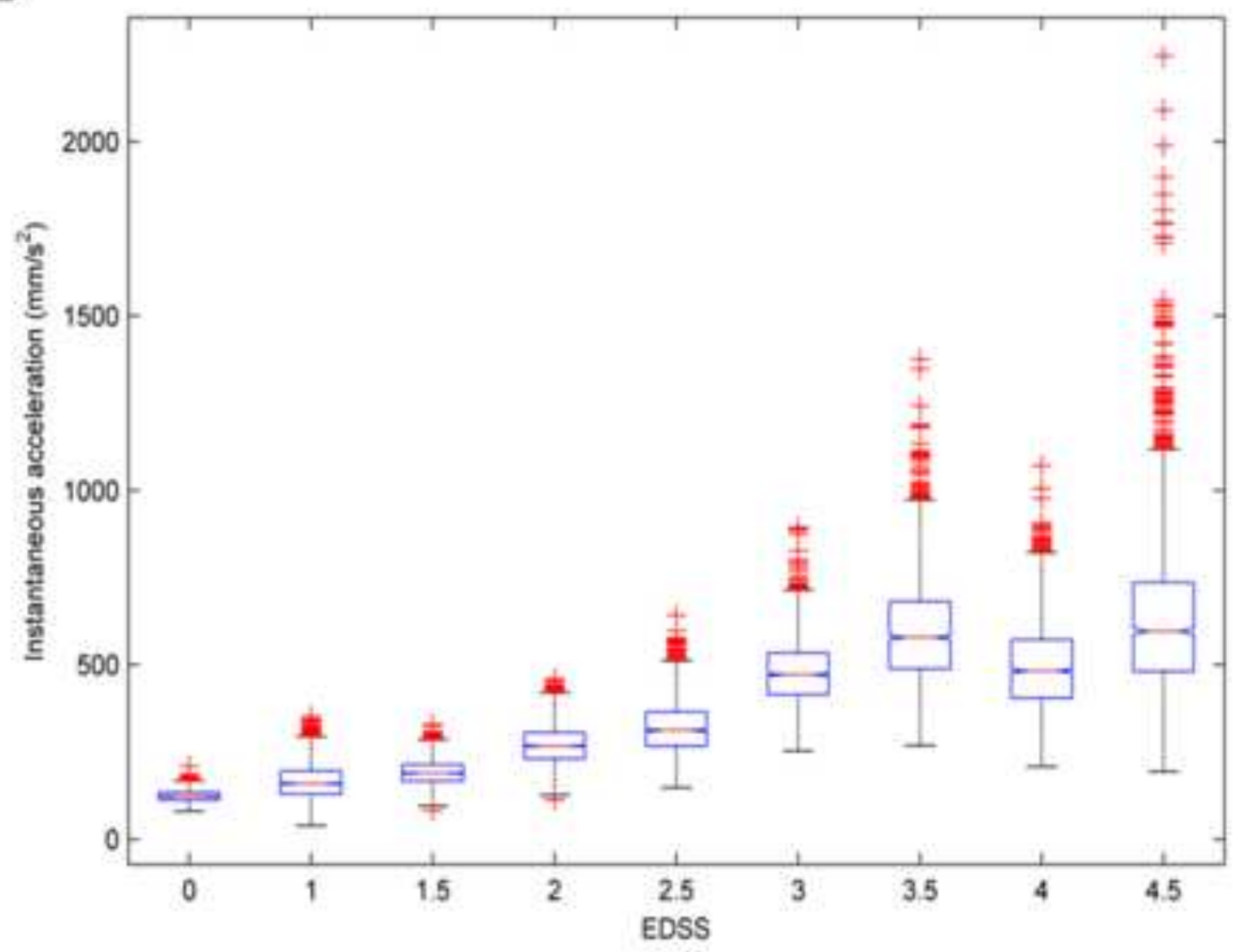


Figure2

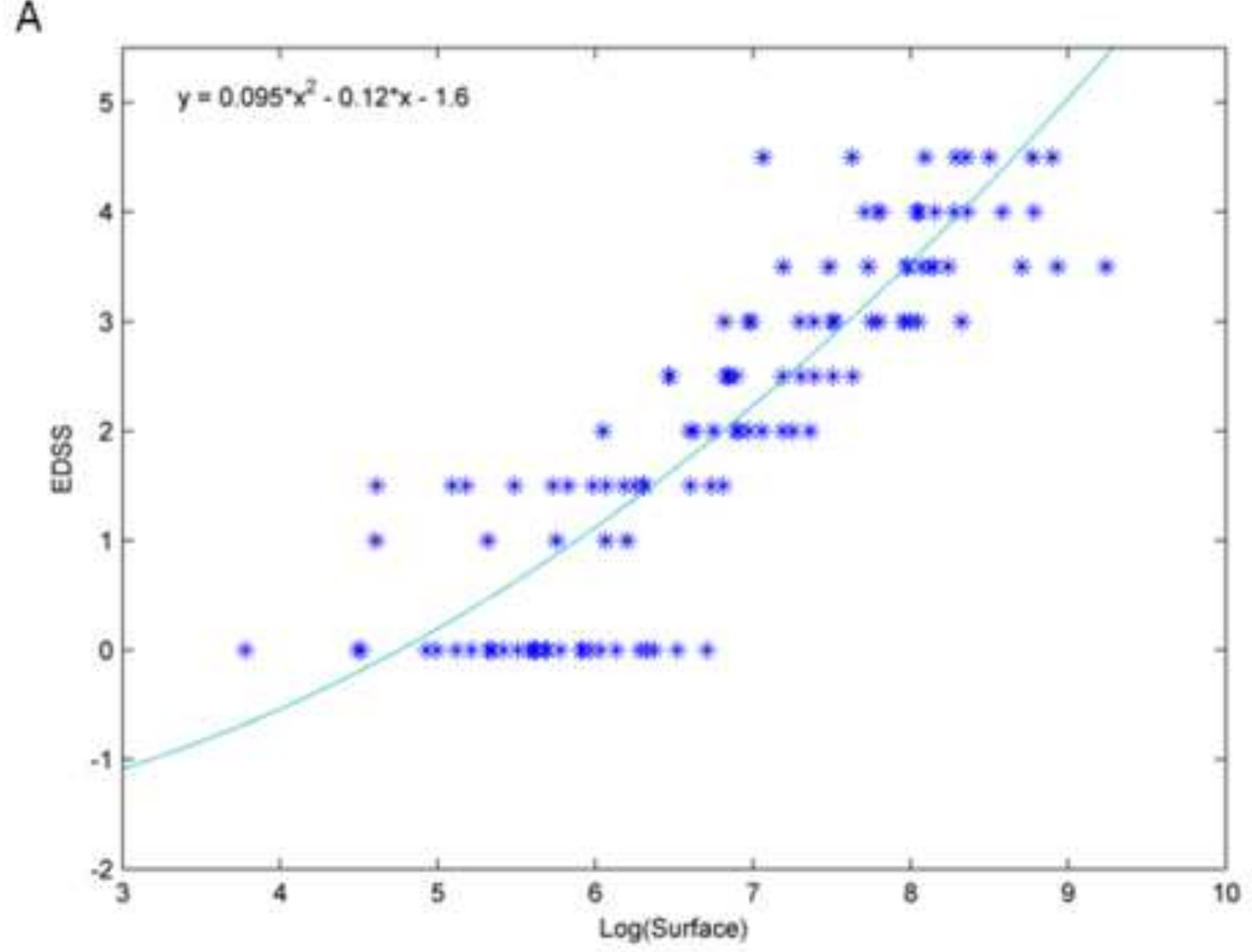

B

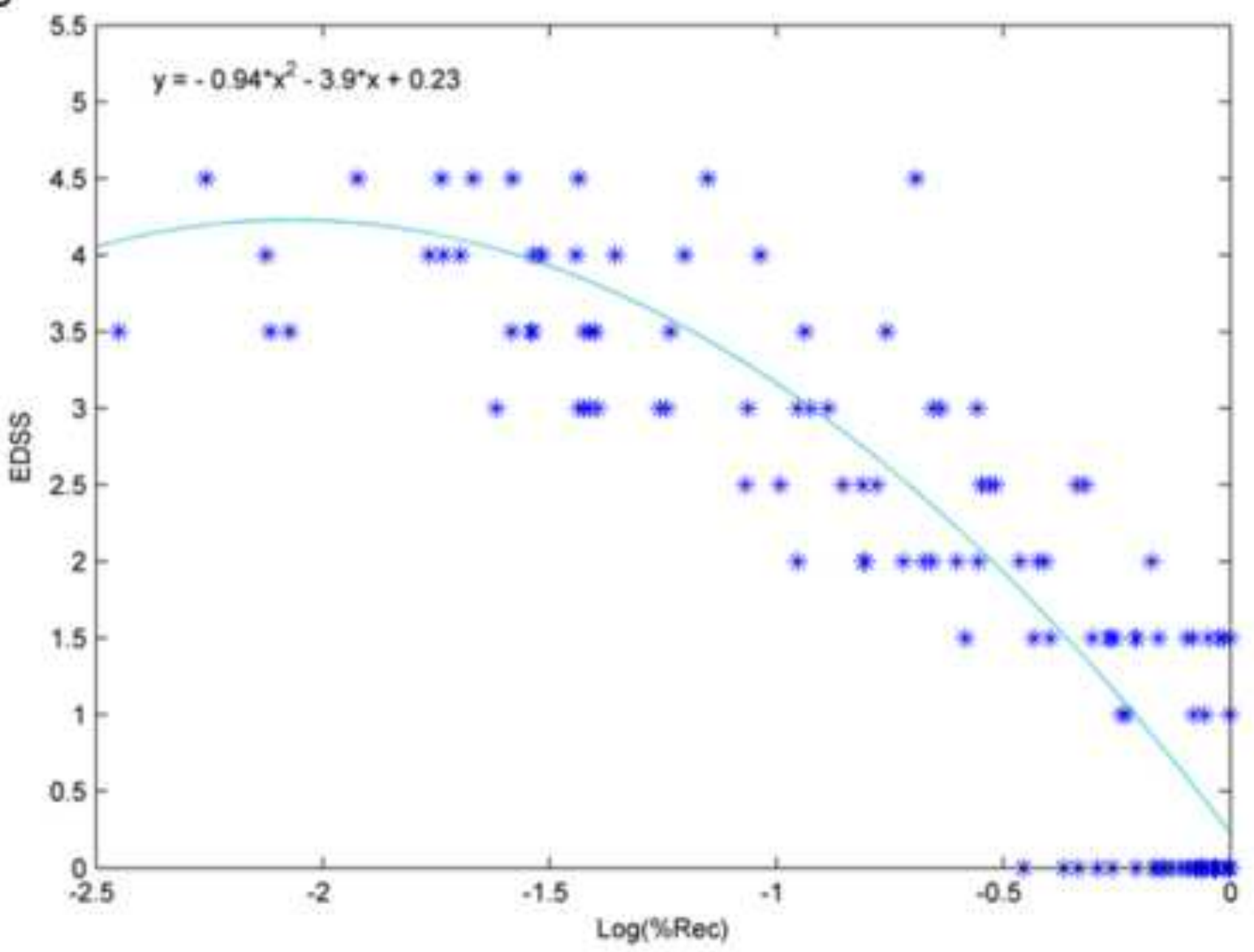

